\title{
Flares from ultracool L dwarfs with Kepler
}

\author{
John E. Gizis ${ }^{1}$, Rishi Paudel ${ }^{1}$, Peter K. G. Williams ${ }^{2}$, Adam J. \\ Burgasser $^{3}$ and Sarah J. Schmidt ${ }^{4}$ \\ ${ }^{1}$ Physics and Astronomy, University of Delaware, Newark, DE, United States, \\ ${ }^{2}$ Harvard-Smithsonian Center for Astrophysics, Cambridge, MA, United States \\ ${ }^{3}$ University of California, San Diego, San Diego, CA, United States \\ ${ }^{4}$ Ohio State University, Columbus, OH, United States.
}

\begin{abstract}
We report on our search for L dwarf flares using NASA's Kepler mission. Spectroscopically confirmedflares were detected with the original Kepler mission from an L1 dwarf stars. We discuss the physicalcharacteristics of these white light flares and compare them to $\mathrm{M}$ dwarf flares. For "habitable zone" planets, the apparent flare brightnesses would be comparable to the most powerful M dwarf flares. Weare monitoring more L dwarfs with the Kepler K2 mission. We discussthe prospect for more detections during the remainder of the K2 mission.
\end{abstract}

Keywords. stars: activity, stars: flare, stars: low-mass, brown dwarfs

\section{Introduction}

As the many contributions to this symposium demonstrate, the Kepler Mission's precision photometry and comprehensive time coverage (Koch et al. 2000) has revolutionized the study of stellar flares (Walkowicz et al. 2011), from A dwarfs (Balona 2012) to G dwarfs (Maehara et al. 2012) to M dwarfs (Hawley et al. 2014). Here we summarize Kepler observations of flares in a nearby L1 dwarf, an ultracool $\left(T_{\text {eff }} \approx 2300 \mathrm{~K}\right)$, fully convective, very-low-mass star smaller then Jupiter.

WISEP J190648.47+401106.8 (W1906+40) was discovered in the original Kepler field midway through the original mission (Gizis et al. 2011). Gizis et al. (2013) summarize the flare observations; Gizis et al. (2015) give a update on the photometric variations over two years. The rotation period is 8.9 hours. W1906+40 is not particularly young; the kinematic and spectroscopic evidence supports an age of several billion years.

\section{Flare Observations}

W1906+40 was observed for one quarter (three months) by Kepler in short cadence mode. In the long-cadence, 30 minute observations, flares are difficult to detect, but the one minute observations allow the flare light curve to be temporally resolved. In all, 21 white light flares were detected; the strongest had an estimated energy of $1.6 \times 10^{32} \mathrm{erg}$. Most show the usual fast-rise-exponential decay shape seen in M dwarf flares (Davenport et al.(2014)). Two show very long decays without a sharp peak; possibly these peaked while on the far hemisphere and then rotated into view, or are due to a more complex magnetic field geometry.

Fortunately, two of the flares occurred during optical spectroscopic monitoring with the Gemini GMOS spectrograph (see Gizis et al. 2013 for full details.) The dim, red photosphere allows the flare's white light continuum component to be clearly detected. A very broad $\mathrm{H} \alpha$ component is also detected at the flare peak. The continuum and broad $\mathrm{H} \alpha$ emission shows the exponential decay seen in the simultaneous Kepler photometry. 
Kowalski et al.(2015) and Kowalski (2015, this volume) model the origins of the white light in flares. Other emission lines, including a narrower $\mathrm{H} \alpha$ component, peak later and decay slowly. Evidently even at the cool temperatures of this L dwarf magnetic reconnection and particle acceleration still occur, resulting in white light flares similar to those in hotter flare stars.

\section{Future Observations}

W1906+40 is no longer observable by the Kepler spacecraft, but we are observing it with Chandra. This will allow us to compare the X-Ray quiscent corona energy budget to the white light energy budget. More generally, are the flares in W1906+40 representative of all early L dwarfs? We have an approved program with the extended Kepler/K2 mission to monitor more ultracool dwarfs in short cadence. Our goal is to determine how the flare rate depends on age, mass, rotation rate, effective temperature or other parameters.

\section{References}

Balona, L. A. 2012, MNRAS, 423, 3420

Davenport, J. R. A., Hawley, S. L., Hebb, L., et al. 2014, ApJ, 797, 122

Gizis, J. E., Troup, N. W., \& Burgasser, A. J. 2011, ApJL, 736, L34

Gizis, J. E., Burgasser, A. J., Berger, E., et al. 2013, ApJ, 779, 172

Gizis, J. E., Dettman, K. G., Burgasser, A. J., et al. 2015, ApJ, 813, 104

Hawley, S. L., Davenport, J. R. A., Kowalski, A. F., et al. 2014, ApJ, 797, 121

Koch, D. G., Borucki, W. J., Basri, G., et al. 2010, ApJL, 713, L79

Kowalski, A. F., Hawley, S. L., Carlsson, M., et al. 2015, Solar Physics, 61

Maehara, H., Shibayama, T., Notsu, S., et al. 2012, Nature, 485, 478

Walkowicz, L. M., Basri, G., Batalha, N., et al. 2011, AJ, 141, 50 